\title{
The power of symmetry
}

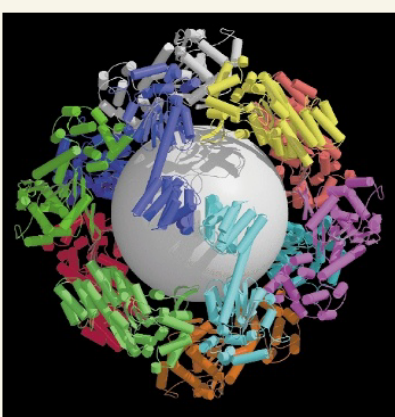

Despite advances in nanotechnology, it has proved difficult to find a robust "recipe" for the creation of a wide range of sophisticated nanomaterials. However, researchers from the University of California, Los Angeles (CA), have found that they can create complex structures such as cages and filaments using the power of protein symmetry (Proc. Natl. Acad. Sci. 98, 2217-2221, 2001). The researchers engineered a gene construct encoding a fusion protein comprising two symmetric selfassembling proteins-the trimeric protein bromoperoxidase and the dimeric M1 matrix protein of the influenza virus-linked by a short stretch of amino acids. Escherichia coli was used to express the recombinant (fusion) protein, which naturally combines with other copies to form a tetrahedral cage of 12 subunits. Using a fusion protein of two dimeric proteins, linked by an even shorter stretch of amino acids, the researchers could create protein filaments that formed bundles. Todd Yeates, who heads the research team, notes that although cages may eventually prove useful for drug delivery, his priority is to design two-dimensional layers that can be used as biosensors, detectors, or molecular sieves.

\section{Unlocking polyketides}

Through extensive metabolic engineering, a team of researchers has produced a strain of Escherichia coli capable of synthesizing polyketides - the chemical building blocks for several blockbuster drugs. The soil bacteria that are currently used to produce polyketides are difficult to culture and are not amenable to genetic manipulation, creating a bottleneck in drug development. Polyketides produced in this manner include erythromycin, the immunosuppressant FK506, and lovastatin, enjoying total annual sales of over $\$ 10$ billion. The researchers introduced genes encoding the three subunits of the polyketide synthase from the soil bacterium Saccharopolyspora erythraea into E. coli (Science, 291, 1790-1792, 2001). They then added genes to generate the precursors for the polyketides, while deleting genes for enzymes that would otherwise break down these cofactors. Using this approach, the scientists produced useful quantities of the core polyketide needed for erythromycin synthesis, and a polyketide not seen before. "We anticipate that $E$. coli could become a useful host for both production of commercial quantities of polyketides as well as to produce novel analogs," says Chaitan Khosla, a researcher at Stanford University and senior author on the paper. Kosan Biosciences (Hayward, CA), which Khosla co-founded, is now working to develop the technology commercially. $A D$

Research News in Brief written by Jackie Barlow, Aaron Bouchie, Alan Dove, and Liz Fletcher.

\section{Cancer's fatal attraction}

Cancers that have spread from their original site-that is, metastasized-are difficult to treat and can be fatal. However, what triggers metastasis, and why secondary tumors arise in some tissues/organs but not others, has long puzzled researchers. Now, Anja Muller and colleagues at Schering-Plough's DNAX Research Institute (Palo Alto, CA) suggest that chemokines could be the fatal cue for metastasis of breast and skin cancers (Nature 410, 50-56, 2001). The researchers found that the chemokine receptor subtypes CXCR4 and CCR7 were upregulated in several lines of breast cancer cells. Furthermore, the ligands for these receptors-CXCL12 and CCL21, respectivelywere present at high concentrations in organs where metastasis commonly occurs (e.g., liver, lymph, and bone). In vitro, the chemokines encouraged cultured breast cancer cells to adopt the invasive characteristics of a metastasizing cancer. Furthermore, when immune-deficient mice were treated with anti-CXCR4 or anti-CCR7 antibodies in vivo, injected human breast cancer cells were prevented from metastasizing to the lungs. Lead author Albert Zlotnik says that it is too early to predict the potential therapeutic value of chemokine inhibitors, although the mouse studies were "promising". "One of the most pressing questions is to investigate how this model applies to other cancers," he says. Skin cancers, for example, express an additional receptor, CCR10.

\section{Virtual walls for microfluidics}

Microfluidic devices could significantly reduce the size and cost of many laboratory assays. However, many biological assays need a gas/liquid interface, limiting the usefulness of current devices in which reagents flow in enclosed channels. To overcome this limitation, Bin Zhao and colleagues from the University of Illinois at Urbana-Champaign (Urbana, IL) have developed a technique for creating devices with "virtual walls" (Science 291, 1023-1026, 2001). The researchers first created a "sandwich" of two glass slides supported by coverslips, coating the inner layers with a hydrophobic self-assembled monolayer (SAM). A photoresistant template is placed on top of the slide, which is then irradiated with ultraviolet (UV) light. The UV light degrades the molecules on the surface of the monolayer, creating a hydrophilic surface. When an aqueous solution is injected between the slides, the fluid flows between the hydrophilic "tracks" on the surface of the slides. Team leader David Beebe says that the next step is to develop a prototype device with which they can carry out a bioassay. The device is particularly well suited for studies of gas-liquid interactions, such as the exchange of substances in lung tissue.

$J B$

\section{Humanizing plantibodies}

Plants offer a cost-effective means for producing recombinant human proteins. However, the therapeutic value of the plant-derived product is often limited by the difference between plant and mammalian post-translational modifications-in particular, glycosylation. This barrier has in part been lowered by the creation of a hybrid/transgenic plant expressing the human version of $\alpha 1,4$-galactosyltransferase (GalT) (Proc. Natl. Acad. Sci. 98, 2899-2904, 2001). Hans Bakker and colleagues at Plant Research International (Wageningen, The Netherlands) genetically modified a tobacco plant to express human GalT. The team then crossed the modified tobacco plant with a plant engineered to manufacture a mouse antibody. The resulting plant-derived antibody ("plantibody") had a glycosylation pattern that more closely resembled a mammalian antibody than any plantibody produced to date. Further modifications are needed to eradicate the remaining plant-specific modifications of the antibodies, namely the attachment of xylose and fucose sugars. Bakker says that the next challenge will be get plants to manufacture sialic acid: "This sugar is required for many [mammalian] serum proteins." This will require adding another five genes. 